\title{
建筑空气环境人因工程学: 问题、思考与探索
}

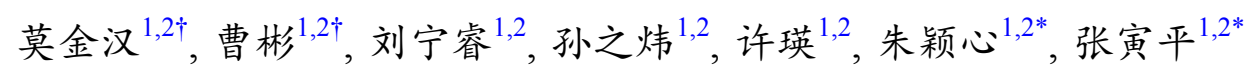

1. 清华大学建筑学院建筑技术科学系, 北京 100084 ;

2. 室内空气质量评价与控制北京市重点实验室, 北京 100084

$\dagger$ 同等贡献

*联系人, E-mail: zhuyx@tsinghua.edu.cn; zhangyp@tsinghua.edu.cn

人 $80 \%$ 以上时间在室内度过，室内空气环境质量对人的 健康、舒适和工作效率有重要影响. 采用节能低碳方式营造 健康舒适的室内空气环境是“健康中国”与“双碳”目标国家战 略的重要内容. 传统的建筑空气环境研究范式存在以下不足: 人的热舒适状况主要基于主观感受调研，人的健康状况主要 基于患病率调研; 空气环境监测参数在种类、时空分布上非 常有限; 传统室内空气热质过程优化主要基于工程热力学和 传热传质学, 前者仅适用于平衡态下的热-功转换过程, 后者 虽有强化概念但缺乏优化概念，在确定建筑环境涉及湿和多 种污染物的非平衡态热-质-功的复杂过程优化时遇到实质性 困难. 为此, 室内空气环境领域的一些重要基础科学问题难 以破解：室内空气环境对人健康、舒适和工效的关键影响因 素及影响机理, 室内空气环境达到目标状态的理想热质过程 确定方法及实现途径. 为克服传统理论局限, 作者基于近年 来的科研探索和思考, 提出了构建建筑空气环境人因工程学 的新思路和新方法: 采用现代技术(如环境和生理信息实时采 集技术、大数据技术和机器学习技术)深人揭示建筑空气环 境与人的关系; 参照建筑节能核心概念“性能因子 (coefficient of performance, COP; 简称能效比)”, 提出了 “健康收益因子 (coefficient of health-benefit, $\mathrm{COH}$; 简称健效比)”和“经济效益 因子(coefficient of economy-benefit, COE; 简称经效比)"; 借 鉴分析力学最小作用量原理, 提出以 $\mathrm{COE}$ 为作用量, 确定健 康舒适、节能低碳建筑环境理想热质过程. 上述思路和方法 还可为研究者建立建筑环境人因工程学提供借鉴.

\section{1 室内空气环境领域回顾和当前的挑战与 机遇}

空气、水和食物是维持人生命的三要素，一般成人三者 日摄人量分别约为 $15 、 2$ 和 $2.3 \mathrm{~kg}{ }^{[1]}$; 人一生中 $80 \%$ 以上的时 间在室内度过 ${ }^{[2]}$. 因此, 室内空气环境质量对人的健康、舒适 和工作效率具有重要影响.

室内(包括建筑室内、交通工具封闭空间内、地下空间 内等)空气环境(indoor air)包括室内气候(indoor climate)和室 内空气质量(indoor air quality)两部分, 前者关注的对象主要

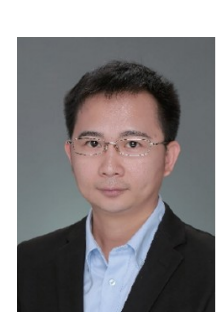

曽金汉清华大学建筑学院副教授, 研 究方向为室内空气污染控制. 2016年获得国 际室内空气学会Yaglou Award，2017年获得 国家自然科学基金优秀青年科学基金项目 资助.

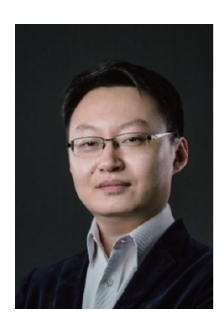

\section{曹㹃清华大学建筑学院副教授, 研究方} 向为建筑热环境与人体热舒适. 2019年获得 美国供热制冷空调学会Ralph G. Nevins Physiology and Human Environment Award, 2020 年获得国际室内空气学会Yaglou Award.

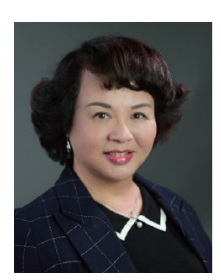

未㖪心 清华大学建筑学院教授, 生态 规划与绿色建筑教育部重点实验室副主任, 教育部高等学校建筑环境与能源应用工程 专业教学指导分委员会主任. 主要从事人体 热舒适与绿色建筑研究.

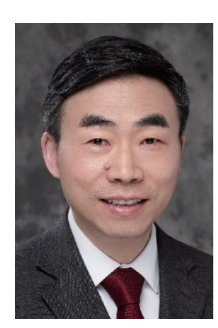

强寅平清华大学建筑学院教授, 室内 空气质量评价与控制北京市重点实验室主 任. 主要从事建筑环境热质过程与健康建筑 研究.

为室内空气的温度、湿度与流动速度, 应满足人们生产、生 活、科学实验和国防等需求; 后者关注的对象主要为空气中 的各种成分及浓度，应保障人的健康、舒适或对目标生物、 
物品(如字画、文物)的影响在可接受范围内. 由于本文主要 讨论室内空气环境因素对人的影响, 不涉及对其他生物类别 和物品的影响, 故只涉及 “人因学”. 为此, 人们首先要认知室 内空气环境对人健康、舒适和工效的影响要素和影响机制, 其次要研发实现的技术途径. 这一过程既不会一䟽而就，也 不会一劳永逸，而会不断受到社会新需求和科技新进展的 “双轮驱动”而持续发展.

室内空气环境的科学认知与现代技术发展历史并不长. Priestley (1733 1804年)发现空气中维持人生存的关键成分 是氧气; Scheele (1742 1786年)和Lavoisier (1743 1794年)发 现空气实际上是一系列气体的混合物, 并揭示了氧气在呼吸 中的作用以及人耗氧量与二氧化碳 $\left(\mathrm{CO}_{2}\right)$ 排放量之间的关系, 由此二氧化碳浓度被当作空气新鲜度(freshness)的量度 ${ }^{[3]}$. Pettenkofer (1818 1901年)注意到人对空气质量的感觉不满 意程度不仅受空气的温度、湿度和 $\mathrm{CO}_{2}$ 浓度的影响, 而且和 人皮肤与呼吸释放的有机物种类及浓度相关. 为此, 他指出: $\mathrm{CO}_{2}$ 本身并不重要, 但可作为空气质量可接受度的指示物(indicator). 1858年, 他在关于居住建筑通风的书中指出, 为保证 人的舒适, 室内 $\mathrm{CO}_{2}$ 浓度不应高于 $1000 \mathrm{ppm}$ (对于 $\mathrm{CO}_{2}, 1 \mathrm{ppm}$ $\left.=1.80 \mathrm{mg} / \mathrm{m}^{3}\right)$, 这个数值后被称为Pettenkofer数 ${ }^{[3]}$. Billings ${ }^{[4]}$ 出版了《通风原理和热舒适》一书, 通风量的确定主要考虑 人体释放的气味和热舒适需求. Yaglou等人 ${ }^{[5]}$ 提出根据人的 气味感知可接受度确定通风量, 被其后的标准广泛应用 ${ }^{[6,7]}$.

20世纪初空气调节(air-conditioning, 简称空调)的发明是 室内空气环境营造的里程碑事件. 为解决印刷车间温湿度不 稳定造成的印刷质量问题, 美国开利(Carrier)博士1902年开 始研发的室内空气温度和湿度调节装置于1907年获得了发 明专利, 他还创建了焓湿图以定量描述热湿处理过程, 奠定 了空调技术应用的设备和理论基础. 随着空调技术和设备的 不断发展, 空调已走出初期的“生产车间”, 逐渐成为满足人们 居住、办公和公共建筑与交通工具等环境温湿度舒适要求 的必备手段, 甚至被新加坡前总理李光耀先生称为 20 世纪最 伟大的发明之一. 与此相伴的是, 室内空气环境热舒适理论 也随之诞生并不断发展. Fanger教授 ${ }^{[8]}$ 于 20 世纪70年代提出 了预测平均评价 (predicted mean vote, PMV)概念, 并以此构 建了描述人体在均匀稳态条件下热舒适程度的PMV方程, 揭 示了 PMV 和室内环境平均辐射温度、空气温度、湿度、流 速、人体活动状况(对应新陈代谢率)与服装热阻间的关联关 系. 后来, 研究者开展了大量研究, 丰富并拓展了热舒适理 论 ${ }^{[9 \sim 13]}$. 需要指出的是, 迄今的热舒适理论还未涉及可能会 影响人热感觉的环境因素, 如光、声、空气质量、环境色彩 和场景. 但生活经验告诉我们, 这些PMV方程中尚未考虑的 因素会对人的热感觉有所影响. 譬如, 房间其他条件相同时, 内表面分别呈现暖色或冷色, 人的热感觉会有所不同. 与此 类似, 感知空气质量(perceived air quality)不仅受空气中成分 和浓度的影响, 还受温度和湿度的影响. 在相同的房间条件
下，温度和相对湿度较低的空气环境，感知空气质量较 高 ${ }^{[14,15]}$.

1952年, 英国伦敦出现严重大气污染(后被称为 Great London Smog), 数周内造成数千人死亡 ${ }^{[16]} ; 20$ 世纪60年代, 发达国家许多工业企业的污染排放和车间空气污染问题开 始引起社会关注. 为此, 美国成立了环保署(Environmental Protection Agency, EPA)和职业安全与健康管理组织(Occupational Safety and Health Administration, OSHA). 室内空气质 量也随后成为EPA和OSHA的关注和控制对象.

20 世纪70年代出现了能源危机，提高能效、减少能耗开 始引发全球关注. 如何实现建筑节能减排成为全世界建筑及 相关领域的共同追求. 为此，以美国为代表的发达国家率先 降低建筑新风量并提高建筑围护结构的气密性. 美国采暖、 制冷和空调工程师学会(American Society of Heating, Refrigerating and Air-Conditioning Engineers, ASHRAE)于1981年修 订的新风量标准就显著降低了新风量 ${ }^{[6]}$. 2019年全球建筑能 耗(主要为采暖和空调能耗)不断攀升, 约占社会总能耗的 $30 \%$, 并排放约 $28 \%$ 由化石能源消耗产生的二氧化碳 ${ }^{[17]}$. 2019年我国建筑运行能耗 11.1 亿tce(碳标准煤当量, 1 tce $=$ $29.3 \mathrm{GJ}), \mathrm{CO}_{2}$ 排放 22 亿吨 ${ }^{[18]}$. 为降低建筑新风的采暖和空调 能耗, 不少国家和地区提高了建筑密闭性要求, 但同时也引 发了因新风量不足而导致的健康问题.

20 世纪下半叶起, 大量人工合成材料和化学品由于性能 优越、价格低廉，开始被广泛用于建筑材料、建筑装饰装修 材料以及各类室内用品中, 其中一些会释放对人体健康有害 的物质, 如挥发性有机化合物(volatile organic compounds, VOCs)和半挥发性有机化合物(semi-volatile organic compounds, SVOCs $)^{[19,20]}$. VOCs中的甲醛、苯已被世界卫生组织 (World Health Organization, WHO) 确定为一级致癌物. 这些材 料引发的室内空气化学污染问题首先出现在美国等发达国 家，强调建筑节能后建筑气密性的增强和新风风量的减少又 加剧了上述问题的严重程度, 引发了病态建筑综合征(sick building syndrome, SBS)、建筑相关疾病(building related illness，BRI)和多种化学物过敏(multiple chemical sensitivity, MCS) 问题. 《柳叶刀》(Lancet) 刊文指出 ${ }^{[2]}, 2019$ 年全球归 因空气中 $\mathrm{PM}_{2.5}$ 和臭氧污染的早死人数达到 667.2 万人. 由于 人在室内外逗留时间比约为 $10: 1$, 因此上述污染暴露主要在 室内. Logue等人 ${ }^{[22]}$ 研究发现, 美国室内空气污染引发的慢性 健康危害导致的以伤残调整生命年(disability-adjusted life years, DALYs)表征的疾病负担为每 10 万人 400 1100 DALYs, 其中 $\mathrm{PM}_{2.5}$ 、丙烯醛、甲醛的贡献率排名前三.

Sundell ${ }^{[23]}$ 指出: “越来越多的科学证据表明“现代疾病”与 “现代暴露'密切相关, 对那些现代才出现的新型化学物质, 人 类还没有足够的时间适应以致产生新的疾病——现代疾病”, 其致病机理尚不清楚, 相关研究非常缺乏, 大多数必须的科 学研究还没有进行, 甚至还没有开始.” 
我国系世界上人口最多的发展中国家，室内空气环境问 题出现的时间比发达国家滞后了约20年. 1978年起我国实行 改革开放, 城镇化和现代化进程非常迅猛, 发达国家约半个 世纪前先后遇到的问题在我国 21 世纪之交前后几乎同时出 现, 且问题更严峻、更复杂. 2003年, 时任总理温家宝同志多 次强调: 在中国, 再小的事乘以13亿都是大事. 更何况室内空 气环境质量对每个人来说都是关乎健康和民生的大事. 其中 一些主要问题包括:

(1) 我国 20 世纪90年代前由于经济发展水平不高, 冬季仅 考虑满足我国秦岭-淮河以北严寒和寒冷地区人们的集中供 暖需求 ${ }^{[24]}$. 随着近年来我国经济水平的不断提高, 夏热冬冷 地区居民的采暖需求也日益增高, 并开始自行使用各种设备 满足这一需求. 但该地区建筑围护结构保温性能欠佳，如何 兼顾该地区的室外气象条件和居民生活习俗，发展与推广合 适的冬季采暖方式及相应的围护结构改善技术，是今后室内 空气环境领域的一项重要任务.

（2）室外大气污染依然严峻. 虽然2012年我国颁布了《环 境空气质量标准》(GB 3095-2012), 其后又采取了系列措施 使大气污染问题逐年缓解, 但目前不少地区 $\mathrm{PM}_{2.5}$.浓度仍未达 到该标准年均浓度 $35 \mu \mathrm{g} / \mathrm{m}^{3}$ 的要求, 更远高于新颁布的WHO 标准年均浓度 $5 \mu \mathrm{g} / \mathrm{m}^{3}$ 的要求 ${ }^{[25]}$. 大气污染物的室外暴露及其 通过建筑通风和渗风进人室内的室内暴露，危害着人们的健 康. 《柳叶刀》指出 ${ }^{[21]}, 2019$ 年中国归因 $\mathrm{PM}_{2.5}$ 和臭氧污染的 早死人数达 184.8 万人, 占全球空气污染早死人数的 $27.7 \%$, 位 居世界各国之首.

（3）室内空气挥发性化学污染依然严重. 1990年以来, 我 国每年新建建筑面积都在 10 亿平方米左右, 既有建筑改造量 近年来也正在持续增加. 无论对新建建筑还是既有建筑改造, 以人造板为主要原材料的建筑装饰装修材料和复合木家具 在室内环境中都被大量使用, 其中一些会释放以甲醛、苯为 代表的VOCs ${ }^{[26]}$, 导致我国一些城市的甲醛、苯等VOCs 污染 比国际上很多国家或地区的城市更严重 ${ }^{[27,28]}$. 此外, 增塑剂、 阻燃剂等SVOCs的健康危害引起研究者关注: 一些SVOCs如 增塑剂邻苯二甲酸酯会导致儿童哮喘 ${ }^{[29]}$ 、男性生殖系统疾 病 ${ }^{[30]}$ 、免疫功能损害 ${ }^{[31]}$ 和女童乳房发育早熟 ${ }^{[32]}$ 等.

(4) 我国不少欠发达农村地区, 平时炊事或冬季采暖采用 劣质煤炭或生物质燃料在室内空间燃烧并缺乏高效排烟系 统, 造成严重的室内空气污染, 危害人们健康, 甚至造成一些 地区譬如云南宣威地区的高肺癌患病率 ${ }^{[33]}$. 很多欠发达地区 的农村炊事取暖引发的室内空气污染问题目前仍未得到根 本性解决 ${ }^{[34,35]}$.

(5) 20 世纪末, 我国室内空气质量问题调研非常有限, 因 此2002年颁布的《室内空气质量标准》(GB/T 18883-2002) 主要参照世界卫生组织或发达国家标准，主控污染物及其阈 值的确定符合我国国情不足，一个显著标志是 $\mathrm{PM}_{2.5}$ 尚未 列人.
（6）很多传染性疾病如流感、肺结核、严重急性呼吸综 合征(SARS)、禽流感、H1N1病毒感染都会通过空气传播, 且主要感染空间在通风不畅的室内环境 ${ }^{[36,37]}$.2020年初全球 新型冠状病毒肺炎(COVID-19)疫情暴发, 给全世界带来巨大 危害, 截至2021年12月28日24时, 全球确诊病例超过 2.81 亿 人，死亡超过 540 万人 ${ }^{[38]}$. 上述传染病给社会带来巨大危害， 也不断引发人们对传染病空气传播的高度关注以及对室内 空气安全的深人思考 ${ }^{[39,40]}$.

我国对空气环境质量高度重视.2016年，中共中央、国 务院颁布《“健康中国”2030规划纲要》, 建筑空气环境健康 是其中的重要内容. 2020年6月, 习近平总书记指出“要推动 将健康融人所有政策，把全生命周期健康管理理念贯穿城市 规划、建设、管理全过程各环节.”2020年9月11日, 习近平总 书记在科学家座谈会上提出四个面向: “坚持面向世界科技 前沿、面向经济主战场、面向国家重大需求、面向人民生 命健康, 不断向科学技术广度和深度进军. ”2021年, 习近平 总书记提出了“双碳”目标国家战略，即我国在2030年实现碳 达峰, 在2060年实现碳中和.

“健康中国”和“双碳”目标国家战略，为建筑环境研究者 带来了机遇和挑战, 甚至会引发科学和技术革命. 抓住机 遇、应对挑战, 需要认知其中的重要基础科学问题, 并分别 从战略层面和战术层面认真思考破解这些问题的新思路和 新途径.

\section{2 室内空气环境领域存在的问题}

在室内环境热舒适领域，亟待解决的社会需求问题为: (1) 在“双碳”目标指引下，如何既能控制建筑能耗和碳排放 的增长, 又能营造出舒适的室内热环境, 满足人们日益增长 的对建筑环境品质的要求? (2) 在“健康中国”战略的背景下, 对室内热环境的营造目标除了实现较高的舒适度, 还应重点 关注健康影响，舒适是否等同于健康? 什么样的室内热环境 营造理念和技术策略有利于保障人体的热健康?

与之相关的关键科学和技术问题主要体现在以下几 方面:

(1) 自然环境与人工环境对人体热舒适影响差异的机理 尚待明晰. 在优良的自然环境中, 人对风、光、声等多种环 境因素的感受往往比人工环境更愉悦. 现有研究通过比较功 率谱密度、湍流度等气流动态特征参数, 已能够从物理层面 揭示自然风和机械风的差异，而这种差异为何会引起人体感 觉的不同, 其机理仍待进一步揭示. 这一问题的解决, 将深化 热环境对人影响机理的认知, 进而通过更科学的建筑设计策 略和设备系统，营造出更接近大自然的室内环境，在不增加 能耗和二氧化碳排放的前提下显著提升人的舒适度.

（2）室内热经历对人体健康的影响机制亟待系统和深人 研究. 现有研究表明, 长时间处于稳定的空调采暖环境中, 会 导致人体热应激调节能力下降，提高病态建筑综合征的发生 
概率 ${ }^{[41,42]}$. 目前, 有不同学者采用皮肤温度、核心温度、血流 量、热应激蛋白、棕色脂肪等多种指标开展人体热生理研 究 ${ }^{[42 \sim 44]}$. 从能够反映人体热健康的关键指标篮选、范围界定, 到深人解释短期热经历和长期热经历对人体热健康的影响 机制, 仍需要开展系统和深人的机理研究. 这一问题的解决, 有助于人们明确室内热环境的健康营造理念，应采用稳定的 “恒态”营造模式, 还是更接近自然的“动态”调节模式.

（3）基于“精准热服务”理念的人体冷热需求识别与调控 技术亟待研发. 传统的空调采暖设备, 通过对流、辐射形式 将大量的冷热量释放到室内空间，其中只有少部分作用到人 体，造成了能量的浪费，也难以满足个体之间差异化的冷热 需求. 未来的室内热环境营造应立足于精准服务理念, 在人 的个体尺度甚至局部实现冷热精准调节, 既有利于提升人员 满意度，又能放宽室内整体环境的温度范围，从而降低空调 采暖能耗. 为了实现“精准热服务”, 首先需对人体冷热需求 进行精准识别, 其次应具备个体尺度或人体局部尺度的冷热 调节装置, 其中涉及基础理论研究与设备研发, 是热舒适领 域的新兴热点.

在室内空气质量方面，亟待解决的社会需求问题为：(1) 我国统一要求的和不同地区、不同建筑类型分别要求的室 内空气污染物应该控什么? 控多严? (2) 室内空气质量控制效 果如何评价? (3) 室内空气污染应该如何节能低碳地防控? （4）如何构建健康增强效果的室内空气环境，譬如具有良好 森林环境的清新空气? (5) 如何节能低碳地综合控制室内空 气热湿环境和空气质量?

以上问题具体表现在:

(1) 很多室内空气单种污染物或多种污染物组合体暴露 剂量与人体效应间的关系即D-R(dose-response)关系尚不清 晰. 以往由于测试技术和成本的限制, 室内空气环境中污染 物监测在污染物种类、浓度测试精度、测试时间跨度和空 间分布上严重不足，难以较为准确地确定室内人群空气环境 中污染物的暴露剂量, 从而难以准确获取室内空气污染暴露 导致的人体效应.

（2）室内空气环境健康增强因素及其健康增强机制不清 晰. 以往研究主要聚焦在降低空气污染物对人健康的负面影 响, 而对如何通过改善室内空气环境质量增进健康则鲜有涉 及. 实际上, 健康建筑不仅是防病建筑, 还应是健康增强建筑. 不明晰室内空气环境健康增强因素及其健康增强机制, 就无 法构建真正的健康建筑.

（3）室内空气环境理想热质过程确定原理与方法亟待研 究. 传统室内空气热湿环境过程优化主要基于工程热力学和 传热传质学，前者仅适用于平衡态下的热-功转换过程分析, 后者虽有强化概念但缺乏优化概念，在确定涉及建筑环境中 水蒸气和多种污染物非平衡态热-质-功的复杂过程优化 (最 节能或最节资)时遇到实质性困难, 难以确定室内空气环境达 到目标状态的理想热质过程及实现途径.
此外, 在健康舒适、低碳节能的建筑空气环境构建过程 中, 会涉及建筑、建材、家具、家电、自动控制等多个行业, 不同行业采用不同的技术术语和标准，常出现“车不同轨、 字不同文”的现象. 譬如，以往在建材行业，材料污染程度仅 用目标污染物“含量”或“释放率”，即单位面积材料的污染物 释放速率表示，但含量高并不意味着释放率高，而释放率受 环境因素(如空气温度、相对湿度、空气流速、承载率)影 响, 实验条件下测得的释放率并不能推广到实际使用条件. 在建筑行业, 室内空气质量设计评价或称预评价需要建材的 释放特性参数(例如, 对于VOCs而言, 包括初始可释放量 $C_{0}$ 、 扩散系数 $D$ 和分配系数 $K$ ), 但建材行业的技术和标准常不涉 及. 这样一来, 建筑室内空气质量预评价成为“少米之炊”, 难 以大规模实施. 建筑室内空气污染负荷指标和空气净化处理 设备的能力指标间也存在技术和标准不匹配的问题，导致空 气净化设备的合理选型和运行优化也难以开展. 实际上，行 业分割导致的问题还很多, 上述问题只是“管中窥豹”而已.

\section{3 克服理论局限和行业分割问题是破解上述 问题的关键}

破解上述问题，不仅需要克服传统理论局限的新思路和 新方法, 还需要学科间的融汇和行业间的贯通. 需秉承“以应 用带基础、以全局统局部、以交叉促创新，以合作求发展” 理念, 把室内空气环境与人的关系作为一个整体性基础科学 问题来对待，从不同方面对这只“大象”进行全面和深人剖析， 避免“盲人摸象”式片面性认知. 在此基础上, 建立室内空气环 境人因工程学, 并在相关行业间贯通应用. 具体思考和建议 如下:

（1）需要通过医工结合破解室内空气环境健康相关的基 础科学问题. 室内空气质量控制涉及工程科学和医学, 两者 在过程特性、关注参数和数学基础方面差异显著, 参见图1. 顺着污染物从源传递到人体的过程，描述参数依次为工程科 学采用的“浓度”、连接工程科学和医学的暴露科学采用的 “剂量”即浓度对暴露时间的积分量、医学采用的“生物标志 物水平”和“疾病程度”[45]. 其中, 工程科学描述的过程一般为 确定性过程, 常用数理方程描述; 医学描述的过程一般为非 确定性过程, 常用概率论和统计学描述. 只有工程科学和医 学的良好合作，才能很好解决建筑环境的健康舒适问题，犹 如两者握手, 才能形成 $H$, 即Health的大写首字母.

(2) 充分利用现代科学和技术手段, 揭示室内空气环境 要素对人的影响程度和影响机制. 如图2所示, 以往受技术手 段局限, 室内空气环境监测参数从种类到数量都非常有限, 基本只监测空气温度和湿度, 很多重要的参数如 $\mathrm{CO}_{2}$ 浓度、 $\mathrm{PM}_{2.5}$ 浓度、甲醛浓度等鲜有实时监测; 人的生理参数获取主 要靠体检, 平时基本没有较为广泛的生理参数监测, 而人的 空气环境热舒适程度主要基于问卷调研, 生理反应指标监测 较少; 健康状况主要依赖于流行病学调研. 由于环境参数和 

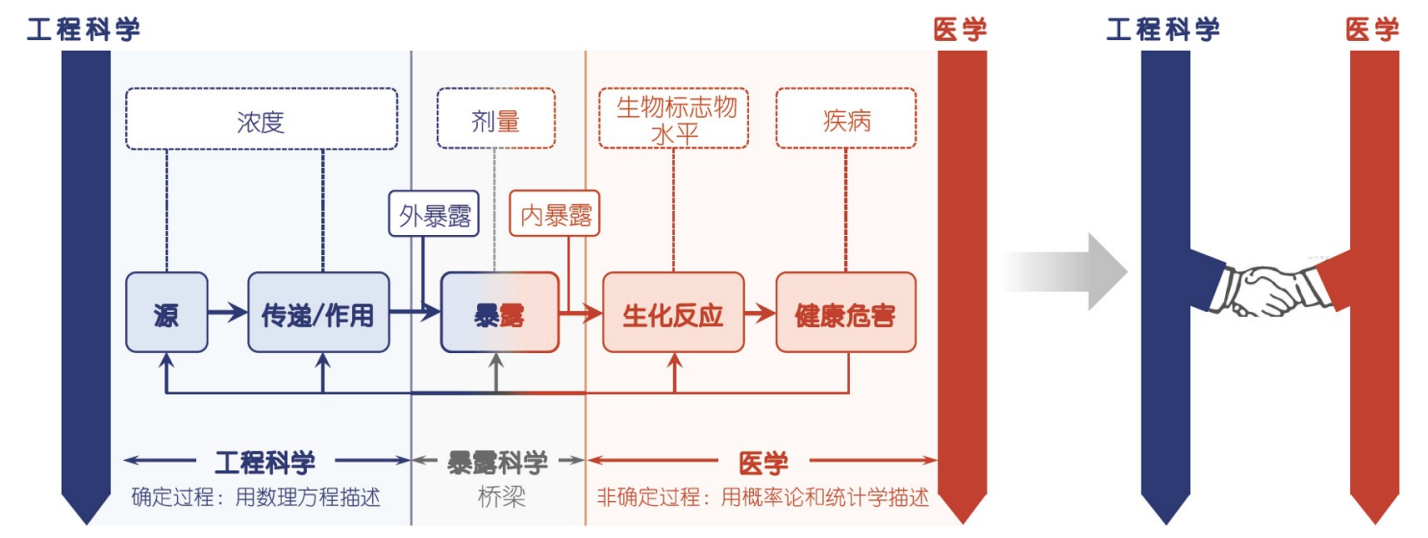

图 1 室内空气污染从源到人的全过程一览

Figure 1 Schematic of the whole process of indoor air pollution control

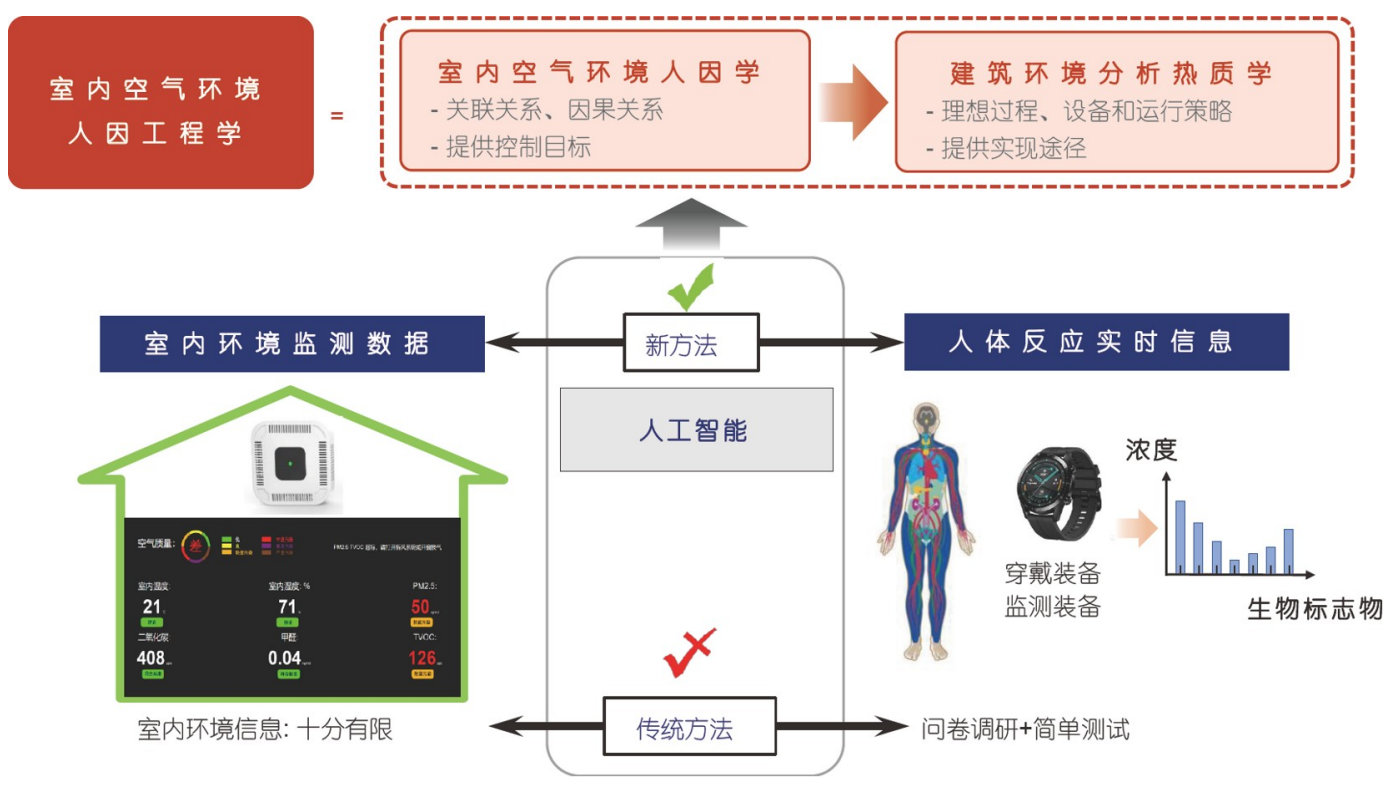

图 2 传统方法局限及建筑空气环境人因工程学特点一览

Figure 2 Schematic of the limitations of traditional approaches and the salient features of "Ergonomics for Indoor Air Environments"

生理参数的种类和数量严重不足, 难以深人认知室内空气环 境对人健康和舒适的影响程度和机制.

近年来, 空气环境参数在线监测技术取得长足进步, 大 量实时温度、湿度、 $\mathrm{CO}_{2}$ 浓度、甲醛、苯系物、总挥发性有 机物即TVOC等污染物浓度探测器问世, 但其中甲醛、苯系 物、TVOC的浓度实时探测器往往价格和准确度难以同时令 人满意, 规模化应用尚有困难. 此外,一些价格适中的可监测 人体生理参数的穿戴设备也应运而生, 可实时监测一些生理 参数如血压(blood pressure, BP)、心率(heart rate, HR)、心率 变异性(heart rate variability, HRV)、血氧浓度、心理压力、 睡眠状况(睡眠时长、深睡眠/线睡眠/快速眼动睡眠/时长及 比例、呼吸质量、醒来次数), 且可无线传输进入云平台. 此 外, 还有大量生理和心理研究型仪器可监测人在不同空气环
境下的生理和心理指标.

在获得室内空气环境参数与人体生理参数的数据后, 如 何通过关联性分析来认知两者之间的定量关系(包括关联关 系和因果关系)? 近年来高速发展的大数据分析技术为我们 破解上述难题提供了手段.

经典统计学是大数据分析坚实的理论基础, 它与确定性 方法不同, 通过概率描述从已观测到的样本数据去推断总体 特征. 统计学方法可以刻画单组或多组样本数据的特征和关 系, 比如描述数据本身的特征(数字特征、拟合优度检验、单 样本假设检验等), 比较数据与数据之间的关系(多样本假设 检验、列联表分析、相关分析等), 或者探索数据内部的结 构(聚类分析、判别分析、主成分分析、多维尺度分析、因 子分析) 等 $^{[46,47]}$. 统计学方法也可以挖掘多个自变量与因变量 
之间的定量关联关系，包括常见的线性回归模型、广义线性 模型(逻辑回归、泊松回归)、方差分析/协方差分析、时间 序列分析和生存分析等 ${ }^{[48,49]}$. 此外, 20世纪末, 统计学领域又 兴起了因果推断理论，来探究事物之间的因果关系而非关联 关系, 并应用到流行病学、生物医学, 甚至近年来的经济 学、社会科学领域中 ${ }^{[50,51]}$.

机器学习则是基于统计学方法, 与数学、计算机等多学 科交叉融合所产生的新研究领域, 本质上基于数据驱动思想, 适用于解决海量数据的预测和分析问题. 机器学习方法大体 可分为监督学习和无监督学习两大类. 监督学习是利用有标 注的数据(既有自变量 $X$, 又有因变量 $Y$ 的数值), 解决分类、标 注、回归等实际问题的一大类方法. 常见的监督学习方法包 括决策树、朴素贝叶斯法、支持向量机、隐马尔可夫模 型、 $k$ 近邻法、期望极大算法(expectation maximization, EM). 无监督学习指的是利用无标注的数据(数据只有多个变量 $X$ 的数值), 解决聚类、降维的问题, 常见方法包括 $k$ 均值聚类、 主成分分析、语义分析和图分析. 机器学习方法最终常转化 为模型超参数的优化问题: 在海量数据驱动下, 利用交叉验 证方法, 以训练集来实现模型学习, 以测试集的损失函数最 小为目标函数, 利用优化理论求解该优化问题 ${ }^{[52]}$.

经典统计学方法与机器学习方法各有特点. 前者涉及的 模型往往有着清晰的物理意义，模型不要求数据量很大，既 可研究关联关系, 也可研究因果关系; 后者要求数据量很大, 其预测结果的精准性往往会高于经典统计学模型. 但由于机 器学习方法常属于黑箱模型, 其解释能力较统计学方法要差, 而且只能研究关联关系, 无法刻画因果关系. 两类大数据分 析方法的比较见表 $1^{[53,54]}$.

采用哪种大数据分析方法, 需要针对不同问题的特点来 选择. 下面举例说明.

应用场景1: 室内空气环境对人体热舒适的影响. 在建筑 室内环境热舒适评价中, Fanger教授 ${ }^{[8]}$ 提出了预测平均评价 PMV指标及评价方法，但他并没有考虑环境色彩、声环境和 光环境等因素对热舒适都可能造成影响. 关于空气质量, 已 有研究表明，人对空气质量的感知与对热环境的感知存在某 种关联. 例如, 温度和湿度对室内空气质量的即时感知和适 应性感知都有显著影响 ${ }^{[55]}$; 在恒定的污染水平下，感知的空 气质量随着空气温度和湿度的升高而下降. 温度和湿度对空 气质量感知的影响随着空气污染程度的增加而减小, 而污染

\section{表 1 两类大数据分析方法的比较}

Table 1 Comparison of two big data analysis methods

\begin{tabular}{ccccc}
\hline $\begin{array}{c}\text { 数据分析 } \\
\text { 方法 }\end{array}$ & 数据量需求 & 模型精度 & $\begin{array}{c}\text { 模型解释 } \\
\text { 能力 }\end{array}$ & 研究关系 \\
\hline 经典统计学 & 中等 & 较高 & 较高 & $\begin{array}{c}\text { 关联关系 } \\
\text { 因果关系 }\end{array}$ \\
机器学习 & 大 & 高 & 较低 & 关联关系 \\
\hline
\end{tabular}

程度对空气质量感知的影响随着空气温度和湿度的增加而 减小 ${ }^{[14]}$, 因此降低室内空气温度和湿度可以显著改善感知空 气质量. 本文作者团队针对室外热环境进行的问卷调研结果 发现, 有大比例被调研者的回答是“室外空气很清新”, 表明人 们日常容易将对热环境和对空气质量的主观感受混杂在一 起. 然而, 上述影响很难通过物理意义清晰的统计模型来描 述. 一个值得探索的途径是, 在未来的研究中结合广泛的室 内环境参数传感监测数据与相应的人群生理指标和主观判 断结果, 利用机器学习方法获得环境参数与人体反应的关联 关系.

应用场景2: 室内空气环境对人健康的影响. 室内空气环 境中暴露因素众多, 包括温度、湿度、多种室内空气污染物 浓度. 识别、篮选出室内空气环境中与人体健康有关的暴露 因素(这需要医学专家参与), 并确定其与预期健康结局的关 联关系, 对保障室内人体健康极为关键, 这也是目前新兴的 暴露组学(exposome)关注的内容. 其难点在于暴露因素的高 维度、暴露因素间的共线性、暴露的时间变异性. 一个值得 探索的途径是, 在未来研究中, 可基于实时监测设备(如具有 生理数据采集功能的手环、手表)获得的长时间人群生理指 标(如血压、心率、心率变异性、精神压力、睡眠长度和质 量、脑电波等)大数据, 采用大数据分析方法, 选出室内空气 环境中对人健康有显著影响的暴露因素．在此基础上，进一 步对因果关系进行深人探究, 这不仅需要毒理学研究者参与, 还需要工程科学和医学研究者合作, 进行暴露参数的干预研 究, 以确定某个(些)暴露因素控制后的健康影响. 具体案例可 参考4.3 小节.

（3）将认识世界和改造世界相关联, 构建室内空气环境 人因工程学. 所谓建筑空气环境人因学, 就是关于室内空气 环境对人健康、舒适和工效的影响规律和机理的学问. 建筑 空气环境的热舒适理论是其重要内涵, 室内空气质量对人的 健康和工效的影响也是其重要组成部分. 相对来说, 前者比 较成熟并自成体系，属于传统建筑环境学的范畴; 而后者涉 及的空气成分、浓度和人的效应关系尚不清晰，且以往被认 为属于医学领域范畴. 我们的观点是: 医学大类中的公共卫 生学和毒理学应该成为建筑环境人因学的理论基础, 就像物 理学、热力学是传热学、工程热力学的理论基础一样, 两者 并不冲突, 而是相辅相成、相得益彰, 参见图2.

认识世界的目的在于改造世界. 客观物理世界中各种自 发运动都符合最小作用量原理. 牛顿三定律是经典牛顿力学 最核心的内容，但分析力学中依据“最小作用量原理”可导出 牛顿三定律, 换言之, 牛顿三定律并不是该理论的出发点, 而 是该理论的导出规律. 它不同于以“力”为核心概念的牛顿力 学，而是以“能”为核心概念的理论体系，不仅反映了与经典 牛顿力学方法论的变化, 而且体现了世界观的不同. 费马以 光程为作用量, 发现光的运动符合“光程最短原理”, 导出了 光学中的反射定律和折射定律. Zhang等人 ${ }^{[56]}$ 以功/热量即 $W /$ 
$Q$ 为作用量, 导出了热力学中的卡诺定理和热力学第二定律.

建筑室内空气环境营造和运维的本质是热量和质量的 传递及其相伴的能量转换, 涉及的热学理论有传热传质学和 工程热力学. 借助于分析力学的思路和方法, 可以构建建筑 环境分析热质学, 它可克服传统热学理论的局限: 热-功转换 过程为平衡态, 传热传质过程仅有强化没有优化, 复杂的非 平衡态热质过程无法优化. 传统热学理论一般采用正问题方 法“由因求果”, 求局部对整体性能的影响, 采用的数学基础为 微积分; 分析热质学采用反问题方法“由果索因”, 求整体性能 对局部的要求, 采用的数学基础为泛函分析 ${ }^{[56]}$.

当我们改造世界时, 我们可选定最主要目标或多目标折 合(最终必须是标量单变量)作为“作用量”，仿照物理世界的 “最小作用量原理”构造过程优化问题，从而获得具体过程的 最优解.

在认知室内空气环境对人的影响规律和机理的基础上, 就获得了人预期空气环境参数范围, 以控制代价为“作用量” 并令其达到极小值，就可确定相关理想热质过程，参见图3. 这种理念和方法就构成了室内空气环境人因工程学的核心 内容, 包括室内空气环境人因学和建筑环境分析热质学两部 分, 参见图2.

将室内空气环境放在室内环境大框架下，可以将室内空 气环境人因工程学的方法拓展, 助力构建考虑室内空气环 境、声环境、光环境相互作用的室内环境人因工程学. 当然, 从理念和设想到成熟的理论还有太多的研究工作要做. 譬如, 室内环境的满意度如何表征和评价? 笔者认为, 可以参照绿 色建筑和健康建筑的分级打分法, 用雷达图评判室内环境热 湿环境、空气质量、声环境、光环境、色彩、空间等方面 的满意度(按人的主观评价)或达标度(按环境参数指标的客 观评价). 而室内环境人因工程学的主要任务就是把室内环境 的某个状态处理到一个人们预设的状态, 并满足代价(能耗或 费用)最小的原则.

利用节能低碳方法构建健康舒适室内环境，不仅需要相
关学科交叉融合, 而且需要相关行业协同攻关. 实际上, 人类 对建筑环境的要求很多, 安全、健康、舒适、美观、节能、 低碳、智能、价格适宜等都是这样要求的具体表述. 要实现 此目标, 首先需要有通用的学术语言. 实际上, 数学语言就是 自然科学领域的“世界语”. 如果所有的过程都可以获得相应 的数字结构，那么多种学科的“方言”也自然可以统一在数字 结构这个“世界语系”之中了. 随着监测技术与设备、互联网 和物联网的迅猛发展, 室内空气环境数据和人生理反应数据 可越来越方便和准确地获得. 与此同时, 机器学习技术、人 工智能技术与相关设备也快速发展，对大数据的分析能力有 了质的提升, 并实现了自学习功能, 这些都为以“机理认知-规 律应用-智能控制”为特点的建筑环境人因工程学的实现提供 了理论、技术和设备基础. 这其中, 还需要贯通相关行业, 实 现全局最优. 在这个全局关联中, 参照建筑节能核心概念“性 能因子 (coefficient of performance, COP; 简称能效比)”, 我们 提出了健康环境核心概念“健康收益因子 (coefficient of health-benefit, $\mathrm{COH}$; =health-benefit/control-cost; 简称健效 比)”和统一优化节能和健康建筑环境控制的核心概念“经济 效益因子(coefficient of economic-benefit, COE; =economicbenefit/economic-cost; 简称经效比)"概念. $\mathrm{COH}$ 可评价室内空 气质量或舒适健康控制代价带来的健康收益, 如果大于 1 , 就 应推荐采用; COE可评价综合控制代价带来的综合经济收益, 如果大于 1 , 就应该推荐采用. $\mathrm{COP}$ 为建筑节能领域的核心概 念, $\mathrm{COH}$ 为建筑环境健康领域的核心概念, $\mathrm{COE}$ 为反映跨领 域综合控制(还可包括声、光、色彩等)途径优劣的核心概 念，它的分子是经济收益，分母是控制代价，均用货币单位， 因此可贯通不同领域, 实现“以全局统局部”的全局最优, 并在 此前提下把经济效益分解到相关领域, 实现“以合作求发展”, 避免某领域追求单领域利益最大化而损坏全局利益.

\section{4 研究案例}

本处案例并非提供涉及问题的完备研究结果，而是说明

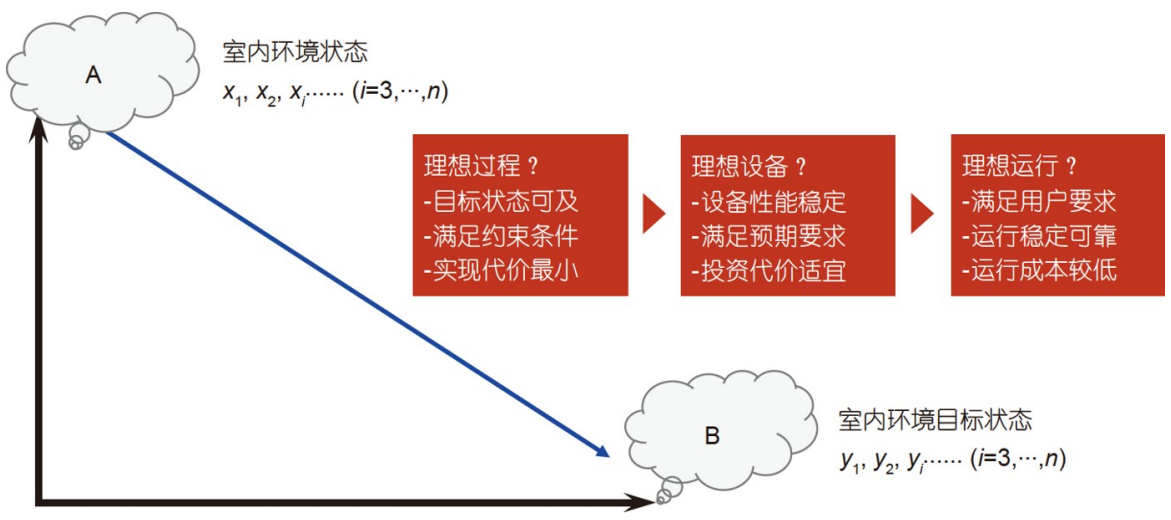

图 3 基于“最小作用量原理”的理想热质过程确定

Figure 3 The determination approach of an ideal heat and mass process by using the least action principle 
如何利用上述概念和方法确定室内空气环境控制方案或策 略. 由于研究工作所限, 本文涉及的内容还非常有限, 希望读 者能通过自身的研究, 突破上述局限, 不断拓展建筑环境人 因工程学的外延和内涵.

\section{1 室内环境综合因素对人体舒适性和工作效率的 影响机理研究}

人体的热舒适性和工作效率受室内声、光、热、空气 质量、色彩、场景等环境因素共同影响. Dong等人 ${ }^{[57]}$ 对 2000 年以来的相关研究进行了系统梳理. 早期的研究大多是针对 两因素之间的交互作用及其对舒适性的影响. 例如: 声、热 研究发现, 热舒适随着噪音提升而降低, 而在合适的温度下, 对声音的舒适感会提升, 恼怒厌烦则会减少; 光、热研究表 明, 明亮度和松弛度受到房间温度的显著影响; 声、光研究 发现, 松弛度受到声音的显著影响 ${ }^{[58 ~ 61]}$. Cao 等人 ${ }^{[62]}$ 在北 京、上海的公共建筑中对温度、照度、噪声级、 $\mathrm{CO}_{2}$ 浓度进 行了实测, 通过回归分析给出了人员总体满意度与上述各环 境参数的定量关系. Huang等人 ${ }^{[63]}$ 通过在办公室中营造不同 噪声级、照度、温度组合的工况进行实验, 发现在办公环境 中, 温度的可接受范围为 $20.9 \sim 30.4^{\circ} \mathrm{C}$, 光的照度为 $300 \mathrm{lux}$, 噪 声在 $49.6 \mathrm{~dB}$ 以下; 同时指出, 温度和噪声舒适度等级对整体 舒适度等级有一票否决效果.

室内环境综合因素对人员工作效率的影响也是目前室 内环境科学领域关注的热点. Hygge和Knez ${ }^{[64]}$ 发现噪音和温 度的交互作用对于长期记忆影响显著, 低噪音、较高温度对 人复述单词能力最有利. Liebl等人 ${ }^{[65]}$ 则发现低等级、高识别 度的噪音会显著削弱短期记忆、理解能力和健康舒适, 动态 照明会导致视觉注意力分散.

上述研究表明，声、光、热、空气质量等因素对人体舒 适性和工作效率的交互影响是普遍存在的, 大部分结论符合 学科既有研究经验, 舒适性参数区间也遵循各学科规律. 但 遗憾的是，现有研究大多集中于同一种类的环境参数之间的 相互影响, 不同种类的环境参数之间的交互影响则很少研究. 例如, Fang等人 ${ }^{[14]}$ 发现热环境对感知的空气质量存在影响, 但人们对空气质量的主观感受是否会影响人体的热感觉则 鲜有涉及. 又比如, 很多学者认为色温的冷暖和照度水平的 高低对人体的热舒适也会有影响, 但却缺乏定量的研究成果 发表. 至于声、光、热、空气质量对环境质量影响的综合评 价, 迄今为止也只有少量的研究发表. 因此, 今后应针对室内 环境多因素交叉影响下的人体舒适性和工效机理进行重点 探究, 确定各环境因素的影响权重, 以指导未来建筑的室内 综合环境性能设计与运行.

\section{2 室内热环境的个体和局部时间与空间控制方式 的节能效果研究}

尽管声光环境对人体的整体舒适感会有影响, 但对人体
舒适性以及建筑能耗影响最大的还是热环境参数. 在常规的 夏季空调环境中, 身着夏装的人在环境温度为 $26^{\circ} \mathrm{C}$ 时会感到 热中性, 这时人体皮肤的平均温度为 $33.7^{\circ} \mathrm{C}$, 与室温的温差为 $7.7^{\circ} \mathrm{C}$. 当室温升到 $30^{\circ} \mathrm{C}$ 时, 环境温度与中性皮温之差为 $3.7^{\circ} \mathrm{C}$, 因传热温差太小而导致偏热不适. 这时, 就需要采用 辅助方式来扩大人体与外界的传热温差. 常规的方法是利用 空调系统把整个房间的室温从 $30^{\circ} \mathrm{C}$ 降到 $26^{\circ} \mathrm{C}$. 目前, 这种空 调降温方式的制冷蒸发温度往往需达到 $5^{\circ} \mathrm{C}$ 以下, 整个空调 系统的 $\mathrm{COP}$ 最高不超过 5.0. 如果对人体局部进行接触式降 温, 根据作者团队研究成果可知, 在室温为 $30^{\circ} \mathrm{C}$ 的环境下, 冷 却座椅表面温度 $26^{\circ} \mathrm{C}^{[66,67]}$ 、降低马甲表面温度 $27^{\circ} \mathrm{C}{ }^{[68]}$ 就可 以维持人体的热中性. 为了把与人体接触的冷却表面维持在 $26 \sim 27^{\circ} \mathrm{C}$, 则需要有一个从 $26 \sim 27^{\circ} \mathrm{C}$ 的表面向 $30^{\circ} \mathrm{C}$ 环境空气转 移热量的热洜. 与现有常规空调系统的制冷温差相比, 其温 差小得多, 系统COP不难达到 5.0, 该方式节能效果显著.

在室温 $30^{\circ} \mathrm{C}$ 的条件下, 一把冷却座椅的椅背和坐垫表面 总供冷量为 $15 \mathrm{~W}$ 就可满足一个人的热舒适需求, 如果其制冷 $\mathrm{COP}$ 为 5.0 , 其电功率仅为 $3 \mathrm{~W}$. 如果办公建筑内人员密度为 $5 \mathrm{~m}^{2}$ (建筑面积)/人，一夏季按工作时间 $880 \mathrm{~h}$ 计，则办公建筑 内单纯冷却座椅的单位建筑面积年电耗为 $0.53 \mathrm{~kW} \mathrm{~h} /\left(\mathrm{m}^{2} \mathrm{a}\right)$.

根据模拟分析, 北京办公建筑夏季空调室内设定温度从 $26^{\circ} \mathrm{C}$ 提高到 $30^{\circ} \mathrm{C}$, 耗冷量下降 $77 \%{ }^{[66]}$. 其原因是不仅室温提 高后空调冷负荷会降低, 而且当室温不超过 $30^{\circ} \mathrm{C}$ 时就不需要 开空调，有效减少了空调开启时间. 如果采用部分负荷时系 统COP变化不大的空调方式, 则相当于空调耗电量也下降 了 $77 \%$.

根据作者团队的现场调查和文献 ${ }^{[69]}$ 的调研数据, 北京市 采用集中和半集中空调的办公建筑夏季单位建筑面积空调 电耗处于 $25 ~ 45 \mathrm{~kW} \mathrm{~h} /\left(\mathrm{m}^{2}\right.$ a) 的范围. 以一个单位建筑面积空 调电耗为 $40 \mathrm{~kW} \mathrm{~h} /\left(\mathrm{m}^{2} \mathrm{a}\right)$ 的某办公建筑为例, 如果将其夏季空 调温度设定值从 $26^{\circ} \mathrm{C}$ 提高到 $30^{\circ} \mathrm{C}$, 则空调电耗就会下降至 $9.2 \mathrm{~kW} \mathrm{~h} /\left(\mathrm{m}^{2} \mathrm{a}\right)$. 加上冷却座椅的电耗 $0.53 \mathrm{~kW} \mathrm{~h} /\left(\mathrm{m}^{2} \mathrm{a}\right)$, 则夏

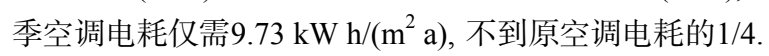

因此，未来开发小温差、小热流密度、高COP的小型热 泵, 将会有力地推动现有的、全空间的室内热环境控制方式 向个体、局部时间和局部空间控制方式的变革，有效地降低 建筑的空调能耗和相应的二氧化碳排放.

\section{3 室内空气污染主控污染物阈值的确定}

我国民用建筑室内空气质量控制依据的标准《室内空 气质量标准》 $\left(\mathrm{GB} / \mathrm{T}\right.$ 18883-2002)中主控污染物没有 $\mathrm{PM}_{2.5}$, 其 修订版补充了此缺陷, 但其室内阈值如何确定仍是非常重要 但意见不一的问题. 完全按世界卫生组织室内空气质量指南 的要求定在年均浓度 $5 \mu \mathrm{g} / \mathrm{m}^{3}$, 不符合我国国情. 为此, 我们提 出按疾病负担和经济收益来确定我国室内空气 $\mathrm{PM}_{2.5}$ 及其他 主控污染物國值的思路. Xiang等人 ${ }^{[70]}$ 基于中国 2015 年339个 
城市的大气 $\mathrm{PM}_{2.5}$ 浓度监测数据, 利用 $\mathrm{PM}_{2.5}$ 已有的 $D-R$ 关系, 估算了各省份人群的室内外 $\mathrm{PM}_{2.5}$ 综合暴露浓度及其导致的 早死人数, 发现当年 25 岁以上人口中, 室外 $\mathrm{PM}_{2.5}$ 导致的早死 人数高达 38.9 万人, 其中 $81 \%$ 系室外 $\mathrm{PM}_{2.5}$ 渗透进人室内造成 的暴露所致. 当室内年均 $\mathrm{PM}_{2.5}$ 浓度控制到 35 和 $10 \mu \mathrm{g} / \mathrm{m}^{3}$ 时, 其导致的早死人数可分别降低 1.4 万人和 16.2 万人. Xiang等 人 ${ }^{[71]}$ 还探讨了不同的室内 $\mathrm{PM}_{2.5}$ 日均限值带来的早死人数和 经济损失减少量, 发现当室内 $\mathrm{PM}_{2.5}$ 日均限值设定在 75 、 $37.5 、 25 \mu \mathrm{g} / \mathrm{m}^{3}$ 时，其导致的早死人数分别可以减少 1.69 万 人、 8.77 万人、 16.55 万人，相应的经济损失可减少 57 亿美 元、294亿美元、552亿美元. 采用类似方法, Xiang等人 ${ }^{[72,73]}$ 评估了中国2015年339个城市的臭氧暴露导致的早死人数, 发现室内外的臭氧暴露造成了 7.08 万人早死，而其中室内的 臭氧暴露贡献了 $59 \%$, 中国现行的室内臭氧时均浓度限值对 减少早死人数作用甚微，建议未来臭氧时均限值设定在 30 ppb以下.

以上研究仅考虑了室内空气污染导致的早死人数影响, 不够全面. 本文以 $\mathrm{PM}_{2.5}$ 的疾病负担水平, 进一步探讨了我国 室内 $\mathrm{PM}_{2.5}$ 浓度阈值确定方法. 我们基于渗透系数法, 估计出 中国各省和直辖市 2017 年室内 $\mathrm{PM}_{2.5}$ 水平 (全国均值为 $33.2 \mu \mathrm{g} / \mathrm{m}^{3}$ ), 并估算出室内外 $\mathrm{PM}_{2.5}$ 暴露造成的疾病负担为 $4.11 \times 10^{4}$ 千人年. 不同的室内 $\mathrm{PM}_{2.5}$ 年均浓度阈值对疾病负担 及相应经济损失的影响如图4所示. 如果将室内 $\mathrm{PM}_{2.5}$ 年均浓 度阈值设定在 $18 \mu \mathrm{g} / \mathrm{m}^{3}$, 其造成的疾病负担可以降低 $20 \%$, 共 降低 $8.22 \times 10^{3}$ 千人年, 相应经济损失可减少 $4.90 \times 10^{3}$ 亿元; 如 果将室内 $\mathrm{PM}_{2.5}$ 年均浓度阈值设定在 $13 \mu \mathrm{g} / \mathrm{m}^{3}$, 其造成的疾病 负担可以降低 $30 \%$, 共降低 $1.22 \times 10^{4}$ 千人年, 相应经济损失可 减少 $7.30 \times 10^{3}$ 亿元.

在实际工程控制中，还需要综合考虑健康收益与控制代 价的平衡，此时可借助经济效益因子 $\mathrm{COE}$ 进行室内空气污染 物浓度阈值的分析. 本文以北京住宅标准卧室 ${ }^{[26]}$ 的 $\mathrm{PM}_{2.5}$ 控制 为例, 估算了不同室内 $\mathrm{PM}_{2.5}$ 年均浓度阈值对 $\mathrm{COE}$ 的影响.

(1) 建筑信息. 北京标准卧室信息 ${ }^{[26]}$, 面积 $16.5 \mathrm{~m}^{2}, 2$ 人, 四季的渗风、通风换气次数已知 ${ }^{[74]}$.

(2) 控制要求. 夏季空调设定温湿度分别为 $26^{\circ} \mathrm{C}, \mathrm{RH}$ $60 \%$, 空调 $\mathrm{COP}$ 为 3.4 ; 冬季集中采暖, 分户热计量, 室内设定 温度 $20^{\circ} \mathrm{C}$; 室内 $\mathrm{CO}_{2}$ 浓度不超过 $1000 \mathrm{ppm}$. 室内 $\mathrm{PM}_{2.5}$ 按设定 年均浓度阈值进行控制。

(3) 控制设备. 采用空气净化器，额定CADR (clean air delivery rate) 为 $360 \mathrm{~m}^{3} / \mathrm{h}$, 售价 1740 元, 5 档调节, 能效 $8.5 \mathrm{~m}^{3} \mathrm{~h}^{-1} \mathrm{~W}^{-1}$, 滤网容尘量 $24000 \mathrm{mg}$, 滤网更换费用 290 元; 净化设备的使用寿命按 10 年计算.

(4) 计算说明. 人员 $\mathrm{PM}_{2.5}$ 暴露估算按每天 $12 \mathrm{~h}$ 在住宅, $4 \mathrm{~h}$ 在室外, $8 \mathrm{~h}$ 在办公室计算; 办公室 $\mathrm{PM}_{2.5}$ 年均浓度按 $25 \mu \mathrm{g} / \mathrm{m}^{3}$ 计算; $\mathrm{PM}_{2.5}$ 暴露导致的疾病负担按照全球疾病负担项目的标 准算法 ${ }^{[21,75]}$ 计算, 对应的 1 伤残调整寿命年经济损失等于北京
当年人均 $\mathrm{GDP}^{[76]}$; 采用空气净化设备后的健康收益根据健康 经济损失降低量估算.

(5) 计算结果. 如图4所示, $\mathrm{COE}$ 随着室内 $\mathrm{PM}_{2.5}$ 年均阈值 的降低而降低. 当室内 $\mathrm{PM}_{2.5}$ 年均阈值高于 $3 \mu \mathrm{g} / \mathrm{m}^{3}$ 时, $\mathrm{COE}$ 均 大于 1 , 说明室内 $\mathrm{PM}_{2.5}$ 控制基本可不考虑能耗和投资代价. 与 此相反, 室外大气 $\mathrm{PM}_{2.5}$ 控制往往代价很高, 因此 $\mathrm{PM}_{2.5}$ 暴露的 防控应重在室内.

实际上，今后亟待提高较为准确的多种典型室内污染物 引发的早死人数和疾病负担的数据量, 这需要医工结合, 做 好顶层设计, 开展有规划和组织的规模性调研, 为我国室内 空气质量控制提供重要基础数据.

\section{4 基于健康风险的室内空气净化方式对人体健康 的影响研究}

《室内空气质量标准》是全国范围内室内空气质量控 制的标准依据，但对某些地区、某些建筑类型和某些新出现 的问题(譬如 4.3 节的负离子空气净化器对健康的影响问题), 该标准尚难以包罗万象. 为此, 需要研究者因地制宜, 及时开 展相关研究，发现问题并对症下药，把这些局部的、早期的 问题解决在“萌芽”阶段. 笔者以一些集中、分散式净化系统/ 设备可能的健康风险问题为例进一步说明上述观点.

(1) 集中净化系统. Day等人 ${ }^{[77]}$ 和Xiang等人 ${ }^{[78]}$ 采用干预 实验和生物标志物评价的方法, 研究了短期使用(2 4周)粗效 过滤器、高效过滤器(high efficiency particulate air filter, HEPA)和静电除尘(electrostatic precipitator, ESP)的复合集中 净化系统对 $\mathrm{PM}_{2.5}$ 和 $\mathrm{O}_{3}$ 暴露及人健康的影响. 研究发现, 相比 于仅使用粗效过滤器, 使用粗效过滤器 + HEPA的组合形式使 得室内 $\mathrm{PM}_{2 .}$ 浓度降低 $80 \%$ 以上, 总暴露降低 $40 \%$ $50 \%$; 然而, 如在系统中增加ESP, 室内 $\mathrm{O}_{3}$ 浓度、 $\mathrm{PM}_{2.5}$ 和超细颗粒物浓度 均会升高; 使用HEPA能有效降低血栓风险，而使用ESP会显

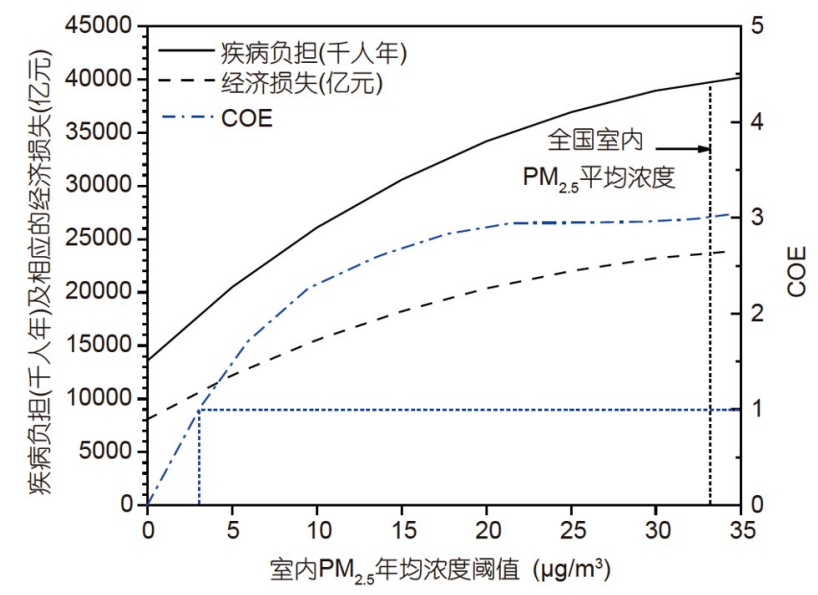

图 4 室内 $\mathrm{PM}_{2.5}$ 年均浓度阈值对疾病负担、经济损失、COE的影响 Figure 4 Effects of indoor $\mathrm{PM}_{2.5}$ thresholds on the burden of disease, corresponding economic costs, and $\mathrm{COE}$ 
著增大血栓风险. 此外, 该研究首次发现低浓度 $\mathrm{O}_{3}$ 暴露(低于 能影响肺功能的暴露浓度)能增大血栓风险, 为改善国内外标 准中室内 $\mathrm{O}_{3}$ 限值提供了科学依据.

(2) 分散式净化设备. Cui等人 ${ }^{[79,80]}$ 招募了 70 名上海大学 生受试者, 开展了短期使用(约 $13 \mathrm{~h}$ ) 空气净化器对室内 $\mathrm{PM}_{2.5}$ 浓度、暴露水平和健康风险(用生物标志物指代)的影响. 研 究发现, HEPA型空气净化器能使室内 $\mathrm{PM}_{2.5}$ 浓度降低70\%, 总 暴露降低约 $40 \%$; 使用净化器的受试者呼吸阻抗 $Z_{5}$ 下降 $7.1 \%$, 总气道阻力 $R_{5}$ 下降 $7.4 \%$, 周边气道黏性阻力 $R_{5} \sim R_{20}$ 下降 $20.3 \%$; 血管性血友病因子(von Willebrand factor, VWF)下降 了 $17.5 \%$, 血栓风险明显下降. 相比HEPA空气净化器, 负离子 空气净化器具有净化能效高、成本低、噪音低等优点, 但其 在降低 $\mathrm{PM}_{2.5}$ 浓度的同时, 会产生大量的负离子. Liu等人 ${ }^{[81]}$ 对 56名北京的健康大学生开展了随机交叉试验, 对学生所居住 的宿舍环境空气污染物浓度进行了监测, 并定期对其生理指 标进行了检测. 结果表明, 负离子连同其在空气中的反应产 物对人体健康可能有不利影响, 需谨慎选用负离子净化器作 为减少 $\mathrm{PM}_{2.5}$ 暴露的手段. 这对在中小学教室具有快速推广态 势的负离子空气净化应用提出了质疑，并呼吁更多的研究者 给予关注和研究.

基于上述研究，教育部《中小学教室空气质量和控制要 求》(JY/T 0636-2021)中提到“臭氧、负离子等会增加全身氧 化应激水平, 因此对于安装这类净化装置应慎重. ”

\section{5 利用“最小作用量原理”确定室内空气温湿度和 污染物浓度综合优化控制策略}

在室内空气质量的控制方面, 以往采用“由因求果”的正 问题思路, 即预设几种净化设备形式和运行策略, 比较能耗和 费用, 在有限方案中寻找“较优”方案, 但无法确定无穷多方案
中的“最优”方案. 同时, 现有空气质量的控制通常只关心空气 质量, 未考虑与室内空气温湿度的综合控制. 因此, 对给定气 候区域和污染条件下的室内空气温湿度和污染物浓度的最佳 综合控制方案, 需要采用以目标为导向的反问题求解方法, 即 以满足室内空气污染物浓度和温湿度要求为约束条件, 以控 制代价(能耗或费用)作为作用量, 以该作用量最小为优化目 标, 求解室内空气温湿度和污染物浓度综合优化控制策略.

Sun等人 ${ }^{[82]}$ 基于上述方法，得到了满足室内 $\mathrm{PM}_{2.5}$ 和 VOC 浓度限值和热舒适要求时能耗最小的新风系统运行策略. 当 有多种室内目标污染物时, 保障室内多种空气目标污染物浓 度中在不同时间段最不易达标的刚好达标, 空气处理过程能

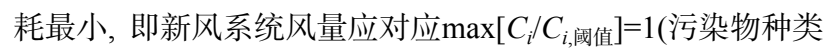
$i=1,2,3, \cdots)$, 见图4.

\section{6 室内空气环境控制健效比COH和经济效益因 子COE分析}

以北京住宅标准卧室 ${ }^{[26]}$ 的 $\mathrm{PM}_{2.5}$ 控制为例, 采用2017年室 外气象数据及 $\mathrm{PM}_{2.5}$ 浓度 ${ }^{[83]}$, 估算了对应的 $\mathrm{COH}$ 和 COE.

(1) 建筑信息. 同4.3节.

(2) 控制要求. 室内 $\mathrm{PM}_{2.5}$ 按设定年均浓度阈值 ${ }^{[84]}$ 进行控 制. 其他同4.3节.

(3) 控制设备. (a) 空气净化器, 参数同4.3节; (b) 新风机, 额定新风量 $170 \mathrm{~m}^{3} / \mathrm{h}$, 售价 6643 元, 5档调节, 能效 $2.6 \mathrm{~m}^{3} \mathrm{~h}^{-1} \mathrm{~W}^{-1}, \mathrm{PM}_{2.5}$ 过滤效率 $95 \%$, 夏季热回收效率 $40 \%$, 冬 季热回收效率 $45 \%$, 滤网容尘量 $6000 \mathrm{mg}$, 滤网更换费用 212 元. 净化设备的使用寿命按 10 年计算.

(4) 计算说明. 同4.3节.

(5) 计算结果. 采用空气净化器, $\mathrm{COH}=\mathrm{COE}=3.08$; 采用新 风机, $\mathrm{COH}=0.71, \mathrm{COE}=0.70$. 因此, 在北京地区的住宅中, 我

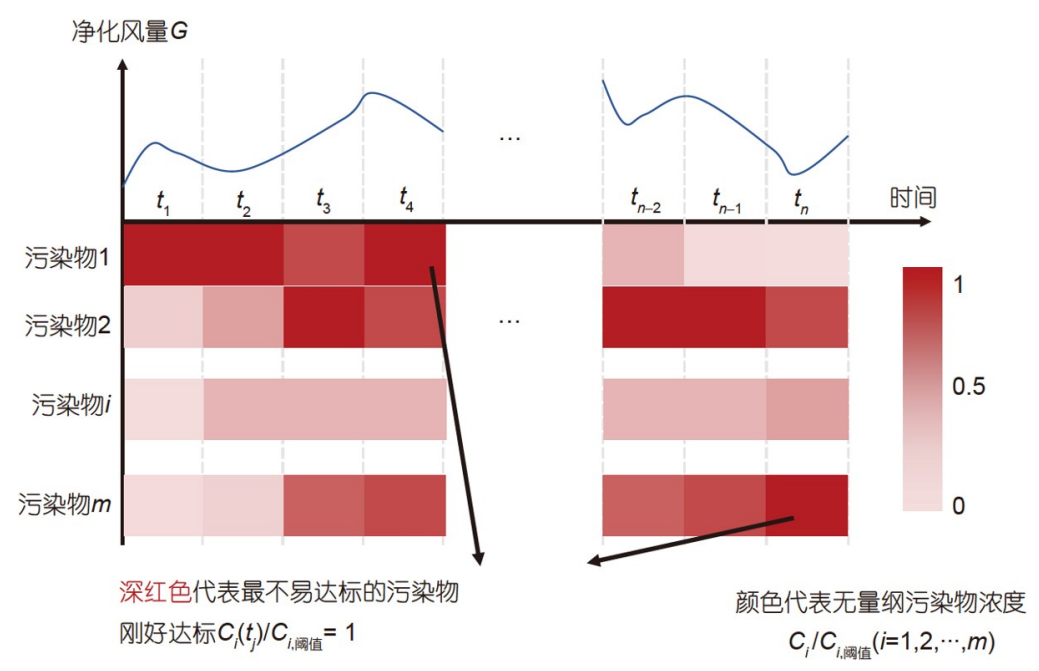

图 5 室内空气质量优化控制原理示意图

Figure 5 Schematic of the optimized control principle of indoor air quality 
们推荐采用空气净化器. 具体计算过程详见补充材料.

\section{5 结论}

为建筑环境领域响应“健康中国”和“双碳”目标国家战

略，应对相应严峻挑战和难得机遇，本文基于近年来相关行
业的科技进步并结合作者自身的科研实践和思考，提出了构 建室内空气环境人因工程学的思路、方法和一些值得研究 的问题, 旨在引发相关领域研究者的研发兴趣, 共同为营造 健康舒适、低碳节能的室内空气环境乃至建筑环境做出不 愧对时代要求的原创贡献.

\section{推葆阅读文献}

1 U.S. Environmental Protection Agency (USEPA). Exposure Factors Handbook. Washington DC: National Center for Environmental Assessment, Office of Research and Development, 2011

2 Klepeis N E, Nelson W C, Ott W R, et al. The National Human Activity Pattern Survey (NHAPS): A resource for assessing exposure to environmental pollutants. J Expo Sci Environ Epidemiol, 2001, 11: 231-252

3 Sundell J. Reflections on the history of indoor air science, focusing on the last 50 years. Indoor Air, 2017, 27: 708-724

4 Billings J S. The Principles of Ventilation and Heating and Their Practical Application. 2nd ed. New York: Engineering and Building Record, 1989

5 Yaglou C P, Riley E C, Coggins D I. Ventilation requirements. ASHRAE Trans, 1936, 42: 133-162

6 Janssen J E. The history of ventilation and temperature control-The first century of air conditioning. ASHRAE J, 1999, 41: 48

7 Persily A. Challenges in developing ventilation and indoor air quality standards: The story of ASHRAE Standard 62. Build Environ, 2015, 91: 6169

8 Fanger P O. Thermal Comfort. Analysis and Applications in Environmental Engineering. Copenhagen: Danish Technical Press, 1970

9 Brager G S, de Dear R J. Thermal adaptation in the built environment: A literature review. Energy Build, 1998, 27: 83-96

10 de Dear R, Xiong J, Kim J, et al. A review of adaptive thermal comfort research since 1998. Energy Build, 2020, 214: 109893

11 Ji W, Zhu Y, Cao B. Development of the predicted thermal sensation (PTS) model using the ASHRAE global thermal comfort database. Energy Build, 2020, 211: 109780

12 Zhu Y, Ouyang Q, Cao B, et al. Dynamic thermal environment and thermal comfort. Indoor Air, 2016, 26: 125-137

13 Zhu Y X, Zhang Y P, Li X T, et al. Built Environment (in Chinese). Beijing: China Building Industry Press, 2016 [朱颖心, 张寅平, 李先庭, 等. 建 筑环境学. 北京: 中国建筑工业出版社, 2016]

14 Fang L, Clausen G, Fanger P O. Impact of temperature and humidity on the perception of indoor air quality. Indoor Air, 1998, 8: 80-90

15 Fang L, Clausen G, Fanger P O. Impact of temperature and humidity on chemical and sensory emissions from building materials. Indoor Air, 1999, 9: $193-201$

16 Bell M L, Davis D L, Fletcher T. A retrospective assessment of mortality from the London smog episode of 1952: The role of influenza and pollution.. Environ Health Perspect, 2004, 112: 6-8

17 United Nations Environment Programme. 2020 Global Status Report for Buildings and Construction: Towards a Zero-emission, Efficient and Resilient Buildings and Construction Sector. Nairobi, 2020

18 Building Energy Research Center of Tsinghua University. Annual Report on China Building Energy Efficiency 2021. Beijing: China Building Industry Press, 2021 [清华大学建筑节能研究中心. 中国建筑节能年度发展研究报告2021. 北京: 中国建筑工业出版社, 2021]

19 Weschler C J. Changes in indoor pollutants since the 1950s. Atmos Environ, 2009, 43: 153-169

20 Weschler C J, Nazaroff W W. Semivolatile organic compounds in indoor environments. Atmos Environ, 2008, 42: 9018-9040

21 Murray C J L, Aravkin A Y, Zheng P, et al. Global burden of 87 risk factors in 204 countries and territories, 1990-2019: A systematic analysis for the Global Burden of Disease Study 2019. Lancet, 2020, 396: 1223-1249

22 Logue J M, Price P N, Sherman M H, et al. A method to estimate the chronic health impact of air pollutants in U.S. residences. Environ Health Perspect, 2012, 120: 216-222

23 Sundell J. Editorial: Climate change is the norm! Why focus on just one pop-problem at a time - energy, mould, sustainability or climate change? When is the time for real indoor air and health science? Indoor Air, 2010, 20: 185-186

24 Ministry of Housing and Urban-Rural Development of the People's Republic of China. Thermal Design Code for Civil Building GB 50176 (in Chinese). 2016 [中华人民共和国住房和城乡建设部. 民用建筑热工设计规范GB 50176. 2016] 
25 World Health Organization (WHO). WHO Global Air Quality Guidelines: Particulate Matter $\left(\mathrm{PM}_{2.5}\right.$ and PM 10$)$, Ozone, Nitrogen Dioxide, Sulfur Dioxide and Carbon Monoxide. Bonn: WHO European Centre for Environment and Health, 2021

26 Liu W W, Zhang Y P, Yao Y, et al. Indoor decorating and refurbishing materials and furniture volatile organic compounds emission labeling systems: A review. Chin Sci Bull, 2012, 57: 2533-2543

27 World Health Organization. WHO Guideines for Indoor Air Quality: Selected Pollutants. Copenhagen, Denmark: WHO Regional Office for Europe, 2010

28 Zhang L, Steinmaus C, Eastmond D A, et al. Formaldehyde exposure and leukemia: A new meta-analysis and potential mechanisms. Mutat Res Rev Mutat Res, 2009, 681: 150-168

29 Oie L, Hersoug L G, Madsen J O. Residential exposure to plasticizers and its possible role in the pathogenesis of asthma. Environ Health Perspect, 1997, 105: 972-978

30 Hauser R, Meeker J D, Duty S, et al. Altered semen quality in relation to urinary concentrations of phthalate monoester and oxidative metabolites. Epidemiology, 2006, 17: 682-691

31 Bornehag C G, Sundell J, Weschler C J, et al. The association between asthma and allergic symptoms in children and phthalates in house dust: A nested case-control study. Environ Health Perspect, 2004, 112: 1393-1397

32 Colón I, Caro D, Bourdony C J, et al. Identification of phthalate esters in the serum of young Puerto Rican girls with premature breast development. Environ Health Perspect, 2000, 108: 895-900

33 He X, Chen W, Liu Z, et al. An epidemiological study of lung cancer in Xuan Wei County, China: Current progress. Case-control study on lung cancer and cooking fuel. Environ Health Perspect, 1991, 94: 9

34 Chen Y, Shen H, Smith K R, et al. Estimating household air pollution exposures and health impacts from space heating in rural China. Environ Int, 2018, 119: 117-124

35 Guan W J, Zheng X Y, Chung K F, et al. Impact of air pollution on the burden of chronic respiratory diseases in China: Time for urgent action. Lancet, 2016, 388: 1939-1951

36 Li Y, Huang X, Yu I T S, et al. Role of air distribution in SARS transmission during the largest nosocomial outbreak in Hong Kong. Indoor Air, 2005, 15: 83-95

37 Yu I T S, Li Y, Wong T W, et al. Evidence of airborne transmission of the severe acute respiratory syndrome virus. N Engl J Med, 2004, 350: 17311739

38 World Health Organization (WHO). WHO Coronavirus (COVID-19) Dashboard. 2021, https://covid19.who.int/

39 Morawska L, Milton D K. It is time to address airborne transmission of coronavirus disease 2019 (COVID-19). Clin Infect Dis, 2020, doi: 10.1093/ cid/ciaa939

40 Morawska L, Tang J W, Bahnfleth W, et al. How can airborne transmission of COVID-19 indoors be minimised? Environ Int, 2020, 142: 105832

41 Cao B, Shang Q, Dai Z, et al. The impact of air-conditioning usage on sick building syndrome during summer in China. Indoor Built Environ, 2013, 22: $490-497$

$42 \mathrm{Yu}$ J, Ouyang Q, Zhu Y, et al. A comparison of the thermal adaptability of people accustomed to air-conditioned environments and naturally ventilated environments. Indoor Air, 2012, 22: 110-118

43 Hanssen M J W, Hoeks J, Brans B, et al. Short-term cold acclimation improves insulin sensitivity in patients with type 2 diabetes mellitus. Nat Med, 2015, 21: 863-865

44 Schrauwen P, van Marken Lichtenbelt W D, Spiegelman B M. The future of brown adipose tissues in the treatment of type 2 diabetes. Diabetologia, 2015, 58: 1704-1707

45 Lioy P, Weisel C. Exposure Science: Basic Principles and Applications. Amsterdam: Elsevier, 2014

46 DeGroot M H, Schervish M J. Probability and Statistics. 4th ed. New York: Pearson Education Inc., 2012

47 Johnson R A, Wichern D W. Applied Multivariate Statistical Analysis. 6th ed. London: Pearson Educational International, 2007

48 Agresti A. Categorical Data analysis. 3rd ed. Hoboken: John Wiley \& Sons Inc., 2013

49 Kutner M H, Nachtscheim C J, Neter J, et al. Applied Linear Regression Models. 5th ed. New York: McGraw-Hill, 2005

50 Pearl J. Causal inference in statistics: An overview. Stat Surv, 2009, 3: 96-146

51 Yao L, Chu Z, Li S, et al. A survey on causal inference. ACM Trans Knowl Discov Data, 2021, 15: 1-46

52 Li H. Statistical Method Learning (in Chinese). Beijing: Tsinghua University Press, 2019 [李航. 统计学习方法. 北京: 清华大学出版社, 2019]

53 Bzdok D, Altman N, Krzywinski M. Statistics versus machine learning. Nat Methods, 2018, 15: 233-234

54 Pearl J. The seven tools of causal inference, with reflections on machine learning. Commun ACM, 2019, 62: 54-60 
55 Fang L, Clausen G, Fanger P O. Temperature and humidity: Important factors for perception of air quality and for ventilation requirements. ASHRAE Trans, 2000, 106: 503-510

56 Zhang Y, Zhang Y, Shi W, et al. A new approach, based on the inverse problem and variation method, for solving building energy and environment problems: Preliminary study and illustrative examples. Build Environ, 2015, 91: 204-218

57 Dong $\mathrm{X}, \mathrm{Wu} \mathrm{Y}$, Chen $\mathrm{X}$, et al. Effect of thermal, acoustic, and lighting environment in underground space on human comfort and work efficiency: A review. Sci Total Environ, 2021, 786: 147537

58 Yang W, Moon H J. Combined effects of sound and illuminance on indoor environmental perception. Appl Acoust, 2018, 141: 136-143

59 Yang W, Moon H J. Cross-modal effects of noise and thermal conditions on indoor environmental perception and speech recognition. Appl Acoust, 2018, 141: 1-8

60 Yang W, Moon H J. Cross-modal effects of illuminance and room temperature on indoor environmental perception. Build Environ, 2018, 146: 280288

61 Yang W, Moon H J. Combined effects of acoustic, thermal, and illumination conditions on the comfort of discrete senses and overall indoor environment. Build Environ, 2019, 148: 623-633

62 Cao B, Ouyang Q, Zhu Y, et al. Development of a multivariate regression model for overall satisfaction in public buildings based on field studies in Beijing and Shanghai. Build Environ, 2012, 47: 394-399

63 Huang L, Zhu Y, Ouyang Q, et al. A study on the effects of thermal, luminous, and acoustic environments on indoor environmental comfort in offices. Build Environ, 2012, 49: 304-309

64 Hygge S, Knez I. Effects of noise, heat and indoor lighting on cognitive performance and self-reported affect. J Environ Psychol, 2001, 21: 291299

65 Liebl A, Haller J, Jödicke B, et al. Combined effects of acoustic and visual distraction on cognitive performance and well-being. Appl Ergon, 2012, 43: $424-434$

66 Yang H C. The effect of the conductive local thermal adjustment on human thermal comfort (in Chinese). Doctor Dissertation. Beijing: Tsinghua University, 2020 [杨贺丞. 传导式局部热调节对人体热舒适的影响研究. 博士学位论文. 北京: 清华大学, 2020]

67 Yang H, Deng Y, Cao B, et al. Study on the local and overall thermal perceptions under nonuniform thermal exposure using a cooling chair. Build Environ, 2020, 176: 106864

68 Zhang Y P, Wang X, Zhu Y X, et al. Special vest as heat sinking for medical garment: Thermal performance and effect of adjusting thermal comfort sense (in Chinese). J HV\&AC, 2003, 33: 58-61 [张寅平, 王馨, 朱颖心, 等. 医用降温服热性能与应用效果研究. 暖通空调, 2003, 33: 58-61]

69 Gao L Y, Quan W, Qin B, et al. Survey and analysis on energy consumption of air conditioners in office buildings in Beijing (in Chinese). Build Technol, 2015, 46: 79-82 [高丽颖, 全巍, 秦波, 等. 北京市办公建筑空调能耗的调查与分析. 建筑技术, 2015, 46: 79-82]

70 Xiang J, Weschler C J, Wang Q, et al. Reducing indoor levels of “outdoor $\mathrm{PM}_{2.5}$ ” in urban China: Impact on mortalities. Environ Sci Technol, 2019, 53: 3119-3127

71 Xiang J, Seto E, Mo J, et al. Impacts of implementing Healthy Building guidelines for daily $\mathrm{PM}_{2.5}$ limit on premature deaths and economic losses in urban China: A population-based modeling study. Environ Int, 2021, 147: 106342

72 Xiang J, Weschler C J, Zhang J, et al. Ozone in urban China: Impact on mortalities and approaches for establishing indoor guideline concentrations. Indoor Air, 2019, ina.12565

73 Xiang J B, Zhang J F, Weschler C J, et al. Effects of tightening standards for indoor ozone levels on associated mortalities in urban China: A population-based modelling study. Lancet, 2018, 392(Suppl): 31-31

74 Hou J, Sun Y, Chen Q, et al. Air change rates in urban Chinese bedrooms. Indoor Air, 2019, 29: 828-839

75 Burnett R T, Pope Iii C A, Ezzati M, et al. An integrated risk function for estimating the global burden of disease attributable to ambient fine particulate matter exposure. Environ Health Perspect, 2014, 122: 397-403

76 Hughes K, Ford K, Bellis M A, et al. Health and financial costs of adverse childhood experiences in 28 European countries: A systematic review and meta-analysis. Lancet Public Health, 2021, 6: e848-e857

77 Day D B, Xiang J, Mo J, et al. Association of ozone exposure with cardiorespiratory pathophysiologic mechanisms in healthy adults. JAMA Int Med, 2017, 177: 1344-1353

78 Xiang J, Weschler C J, Mo J, et al. Ozone, electrostatic precipitators, and particle number concentrations: Correlations observed in a real office during working hours. Environ Sci Technol, 2016, 50: 10236-10244

79 Cui X, Li F, Xiang J, et al. Cardiopulmonary effects of overnight indoor air filtration in healthy non-smoking adults: A double-blind randomized crossover study. Environ Int, 2018, 114: 27-36 
80 Cui X, Li Z, Teng Y, et al. Association between bedroom particulate matter filtration and changes in airway pathophysiology in children with asthma. JAMA Pediatr, 2020, 174: 533-542

81 Liu W, Huang J, Lin Y, et al. Negative ions offset cardiorespiratory benefits of $\mathrm{PM}_{2.5}$ reduction from residential use of negative ion air purifiers. Indoor Air, 2021, 31: 220-228

82 Sun Z, Wang Q, Meng C, et al. New approach to determine the optimal control of fresh air systems in urban China residences. Build Environ, 2022, 207: 108538

83 China National Environmental Monitoring Center. 2021, http://106.37.208.233:20035/

84 Wang Q Q, Wang Y W, Meng C, et al. Assessment Standard for Healthy Building (T/ASC 02-2021) (in Chinese). Beijing: Standards of Architectural Society of China, 2021 [王清勤, 王有为, 孟冲, 等. 健康建筑评价标准T/ASC 02-2021. 中国建筑学会标准. 北京: 中国建筑工业 出版社, 2021]

\section{补充材料}

附录 $\mathrm{COH}$ 和 $\mathrm{COE}$ 的计算说明

本文以上补充材料见网络版csb.scichina.com. 补充材料为作者提供的原始数据, 作者对其学术质量和内容负责. 


\title{
Ergonomics for indoor air environments: Problems, reflections and investigations
}

\author{
Jinhan $\mathrm{Mo}^{1,2 \dagger}$, Bin $\mathrm{Cao}^{1,2 \dagger}$, Ningrui Liu ${ }^{1,2}$, Zhiwei Sun ${ }^{1,2}$, Ying $\mathrm{Xu}^{1,2}$, Yingxin $\mathrm{Zhu}^{1,2^{*}}$ \& \\ Yinping Zhang ${ }^{1,2^{*}}$ \\ ${ }^{1}$ Department of Building Science, School of Architecture, Tsinghua University, Beijing 100084, China; \\ ${ }^{2}$ Beijing Key Lab of Indoor Air Quality Evaluation and Control, Beijing 100084, China \\ $\uparrow$ Equally contributed to this work \\ *Corresponding authors, E-mail: zhuyx@tsinghua.edu.cn; zhangyp@tsinghua.edu.cn
}

People spend over $80 \%$ of their time indoors. Indoor environmental quality has a significant impact on human health, comfort and productivity. Applying energy-efficient and low-carbon methods to create a healthy and comfortable indoor environment is strongly requested by the national strategies of "Healthy China" and "Peak Carbon Dioxide Emissions and Carbon Neutrality". The traditional paradigm of studying indoor air environments has the following limitations: Human thermal comfort information mainly comes from questionnaire surveys; human health information is mainly based on the investigations of morbility and mortality rate; indoor air parameters that are monitored are very limited regarding their types and spatial and temporal distributions; the traditional method to optimize the heat and mass transfer processes of indoor air is based upon engineering thermodynamics and heat and mass transfer, in which the former is only applicable to the heat-work conversion processes under equilibrium states, while the latter lacks the concept of optimization and thus encounters substantial difficulties when dealing with complex non-equilibrium processes involving heat, mass, and work. Due to these intrinsic limitations, the following fundamental problems cannot be solved: The key influencing factors and mechanisms of indoor air environments on human responses have not been well identified and interpreted; and the ideal heat and mass transfer processes for a desired indoor air environment and the corresponding optimized engineering approach have not been determined. To address these problems, we put forward a framework of "Ergonomics for Indoor Air Environments". It is based upon modern technologies (e.g., online sensing technology for indoor air parameters and human responses, and big data collecting and analyzing technologies, including machine learning). By referring to the key parameter in the building energy efficiency field, COP (coefficient of performance), we put forward two new parameters, $\mathrm{COH}$ (coefficient of health-benefit) and $\mathrm{COE}$ (coefficient of economy-benefit). The theory can identify an ideal heat and mass transfer process by using the principle of least action and taking COE as the index of "action", where the "ideal" means that the target indoor air environment can meet all healthy and thermal comfort requirements while the economic cost is minimal. This approach is also helpful for researchers to develop Ergonomics for Indoor Air Environments.

building environment, thermal comfort, indoor air quality, analytical heat and mass transfer, big data

doi: 10.1360/TB-2022-0019 